\title{
Computer vision syndrome prevalence according to individual and video display terminal exposure characteristics in Spanish university students
}

Short running title: CVS prevalence in Spanish university students

Natalia Cantó-Sancho a, b, Mar Sánchez-Brau a, *, Belén Ivorra-Soler c , Mar SeguíCrespo ${ }^{\mathrm{c}, \mathrm{d}}$

${ }^{a}$ Doctoral Programme in Health Sciences, University of Alicante, San Vicente del Raspeig, Alicante, Spain

${ }^{b}$ Department of Community Nursing, Preventive Medicine and Public Health and History of Science, University of Alicante, San Vicente del Raspeig, Alicante, Spain

${ }^{c}$ Department of Optics, Pharmacology and Anatomy, University of Alicante, San Vicente del Raspeig, Alicante, Spain

${ }^{d P}$ ublic Health Research Group, University of Alicante, San Vicente del Raspeig, Alicante, Spain

*Corresponding author: Mar Sánchez-Brau. Doctoral Programme in Health Sciences, University of Alicante, San Vicente del Raspeig, Alicante s/n, Spain. Email: mdmsb7@gmail.com

Financial support: This research received no specific grant from any funding agency in the public, commercial, or not-for-profit sectors.

Disclosure of interest: None of the authors have any proprietary interests or conflicts of interest related to this submission

This article has been accepted for publication and undergone full peer review but has not been through the copyediting, typesetting, pagination and proofreading process, which may lead to differences between this version and the Version of Record. Please cite this article as doi: 10.1111/ijcp. 13681

This article is protected by copyright. All rights reserved 
MISS NATALIA CANTÓ-SANCHO (Orcid ID : 0000-0002-4310-9685)

Article type : Original Paper

\title{
Computer vision syndrome prevalence according to individual and video display terminal exposure characteristics in Spanish university students
}

\begin{abstract}
Aim: To estimate the prevalence of computer vision syndrome (CVS) in university students and its relationship with sociodemographic and optical correction factors and exposure to video display terminal (VDT).
\end{abstract}

Methods: This cross-sectional study included 244 Spanish university students who responded to an anamnesis, a VDT exposure questionnaire and the Computer Vision Syndrome Questionnaire (CVS$\mathrm{Q}^{\odot}$ ). A descriptive analysis was performed and the prevalence of CVS was calculated. Logistic regression models were used to measure the association between CVS and the variables studied.

Results: The mean age was 20.7 years ( $\mathrm{SD}=2.1$ ), $57 \%$ were women, $78.3 \%$ used VDTs $\geq 2$ hours/day to study. The prevalence of CVS was $76.6 \%$, and the most frequent symptoms were headache and itching. In the crude analysis, being a woman, using glasses daily and to study, and a longer VDT use to study and in total were associated with a higher prevalence of CVS; while in the older group, the prevalence was lower. In the multivariate model, VDT use to study was associated with a greater probability of CVS (aOR: 3.43; 95\%CI: 1.03-11.42), and being between 22-29 years was associated with a lower probability of it (aOR: 0.36 ; 95\%CI: $0.15-0.89$ ).

Conclusion: CVS has a high prevalence among Spanish university students. The most affected are the younger ones and those who use VDTs for longer hours to study. It is essential to continue investigating the influence of the type of tasks conducted with VDTs on CVS and thus to establish the preventive measures to reduce this syndrome.

Keywords: computer vision syndrome; university students; computer terminals; prevalence; crosssectional studies; Spain

This article is protected by copyright. All rights reserved 


\section{What's already known about this topic?}

International studies have documented an elevated CVS prevalence in university students due to their high exposure to VDTs.

The limitation of these studies comes from the use of non-validated ad hoc questionnaires to measure CVS, which are focused on individual symptoms. This causes imprecise definitions and does not allow comparing the findings between studies.

Since most of these studies were conducted in Asian countries, there is a knowledge gap about CVS prevalence in European university students.

\section{What does this article add?}

A validated questionnaire to measure CVS as a global construct $\left(\mathrm{CVS}-\mathrm{Q}^{\odot}\right)$ has been used for the first time in a sample of Spanish university students from 44 degree programs.

No previous studies have differentiated the purpose of using VDTs, such as the hours spent studying or leisure, nor if this has different effects on the CVS. Our results suggest that more-demanding cognitive tasks carry a greater risk of experiencing CVS, the most affected being the ones who use VDTs for longer hours to study. 


\section{Introduction}

In recent decades, the development and use of new information and communication technologies (NICT) have become widespread throughout the world, becoming indispensable in personal, professional and academic environments [1]. In the field of education, the use of video display terminals (VDTs), such as computers, laptops, tablets, smartphones and ebooks, is increasingly common among university students since these devices are useful for quickly finding and storing information and for conducting academic or research work. This widespread use has generated a change in the teaching-learning process given that digital devices provide options that improve conventional teaching, thus promoting their use [2-4].

Various published studies report different VDT exposure data. The study by Mowatt et al., [2] indicates that approximately $40 \%$ of students at a university in Jamaica used computers for more than 6 hours a day and that studying is the main reason for their use in $51.3 \%$ of cases. Two studies conducted among university students in Saudi Arabia reported disparate exposure data; while Al Rashidi et al., reported that $36.4 \%$ of students used a computer more than 4 hours a day [4], Al Tawil et al., reported higher exposure, since approximately $50 \%$ of the students used computers more than 5 hours a day [5]. Other studies conducted at universities in Nepal [6], Romania [7] and Malaysia [3], reported that 6.7\%, $25.5 \%$ and $26.2 \%$ of students, respectively, used VDTs more than 5 hours per day and that approximately $60 \%$ of students took regular breaks $[3,6]$. There are no known studies published on the Spanish or European university student population's exposure to digital devices that permit comparisons with data from other countries. Such figures would be interesting given that the socioeconomic development of the context may influence the degree of implementation of NICT in teaching-learning processes and ultimately the use of VDTs by students in their day-to-day lives [8].

The use of digital devices leads to problems related to visual health, musculoskeletal disorders and psychosocial risks. Among the former, computer vision syndrome (CVS), also known as digital eye strain, visual fatigue or asthenopia [1,7,9-11], is a set of ocular symptoms (such as dryness, itching and redness), visual symptoms (such as blurred or double vision and eyestrain) and even headache, which are produced by the prolonged use of VDTs [12-14]. 
Studies indicate that up to $90 \%$ of VDT users have visual problems $[15,16]$, that these symptoms transiently affect almost 60 million people worldwide and that 1 million new cases occur annually [17].

In the university population, several studies on CVS have been carried out in Malaysia [3,17], Saudi Arabia [4,5], the United Arab Emirates [1], Jamaica [2], Nepal [6], Romania [7] and India [18]. The figures vary by country of origin, although all of these studies show an elevated prevalence of CVS. Reddy et al., [3] from a sample of students from different universities in Malaysia, using an ad hoc 12-symptom questionnaire, obtained a prevalence of approximately $90 \%$. However, in the study by Logaraj et al., [18] among students in the final year of medicine and engineering, using an ad hoc 6-symptom questionnaire, the prevalence of CVS was approximately $80 \%$. Both studies considered the students to be having symptoms of CVS if they experienced at least one symptom during/following the use of computer. Among the symptoms most frequently reported by students are eyestrain, with a prevalence greater than $86 \%$ [7,17], headache, with prevalences ranging between $19.7 \%$ and $66.5 \%$ $[1,3,5]$, and blurred vision, dryness and tearing, among others [6]. The results indicate that sex, factors related to VDT exposure, such as hours of use or taking breaks, and the use of optical correction may influence the prevalence of CVS [1-3,5-7,18]. However, the main limitation of these studies is the use of $a d$ hoc questionnaires to measure CVS that have not been validated, and which on numerous occasions did not provide information on the syndrome as a global construct but focused instead on different individual symptoms, with imprecise CVS definitions.

In 2015, Seguí et al., [19] developed and validated a self-administered questionnaire in Spanish to evaluate the CVS (Computer Vision Syndrome Questionnaire, CVS- $Q^{\odot}$ ). This questionnaire facilitates the conduct of quality studies to estimate the prevalence of CVS in the general population and specifically in susceptible groups, e.g. the university population. The CVS- $\mathrm{Q}^{\odot}$ has good psychometric properties derived from the Rasch Analysis, with sensitivity and specificity values of $75.0 \%$ and $70.2 \%$, respectively. This questionnaire has been used in Spanish workers [20-23] and its translation, cultural adaptation and validation into different languages is also being carried out [24].

In the absence of CVS studies in European countries, and particularly in Spain, this study was proposed with the aim of estimating the prevalence of CVS in a sample of Spanish university population and determining its relationship with sociodemographic and optical correction factors and exposure to digital devices. 


\section{Methods}

\section{Design, target population and ethical aspects}

A cross-sectional study was conducted on students at the University of Alicante (UA), Spain, enrolled in undergraduate and master's degrees during the academic year 2018/2019, a population that amounted to a total of 23,827 students.

To calculate the size of a representative sample of the study population, the sample size calculator GRANMO version 7.12 was used. It was calculated that a sample of $n=244$ individuals was enough to estimate with a confidence level of $95 \%$ and an accuracy of $+/-5$ percentage units and a predictable population percentage of $80 \%$; these values were estimated after a previous pilot study on 40 students who attended routine visual check-ups at the UA Optometric Clinic during the 2 months prior to the study. The study was conducted in April 2019 by visiting the different campus faculties (to ensure the inclusion of students from various degree programs), and participants were selected using a purposive sampling.

All students received a study fact sheet and signed an informed consent form. The study was approved by the Ethics Committee (UA-2019-04-12) and was conducted following the standards of Good Clinical Practice and international ethical principles applicable to human research according to the latest revision of the Declaration of Helsinki. In addition, protection of personal data and the guarantee of digital rights were taken into account.

\section{Information collection and procedure}

After participants agreed to participate in the study, they responded to two questionnaires.

First, an anamnesis and exposure questionnaire was designed ad hoc and was always administered in a guided manner by the same researcher. In this questionnaire, sociodemographic (sex, age and university degree), ocular health (ocular disorders, pharmacological treatment and ocular surgery), current optical correction (use of glasses and/or contact lenses) and VDT exposure (daily hours of use of electronic devices for study, both inside and outside the classroom, and leisure purposes) information was collected.

Ocular disorders at the time of the study and general or ocular pharmacological treatment for the preceding month were asked about. Specific examples such as strabismus, amblyopia, conjunctivitis, glaucoma were indicated, emphasizing that ocular disorders did not refer to refractive errors. 
Second, the CVS-Q ${ }^{\odot}$ [19] was administered. This questionnaire evaluates the frequency (never, occasionally and often or always) and intensity (moderate or intense) of 16 ocular and visual symptoms related to the VDT use. These symptoms are burning, itching, feeling of a foreign body, tearing, excessive blinking, eye redness, eye pain, heavy eyelids, dryness, blurred vision, double vision, difficulty focusing for near vision, increased sensitivity to light, colored halos around objects, feeling that sight is worsening and headache. The frequency and intensity data are recoded to calculate the severity of each symptom, which results in a total score. Total scores $\geq 6$ indicate that the subject suffers CVS.

\section{Statistical analysis}

A descriptive analysis of all study variables was performed. Absolute frequency and percentage were calculated for categorical variables. For continuous variables, the mean and standard deviation (SD) and the minimum and maximum were obtained. The prevalence of CVS was calculated for each of the variables and categories. To determine whether there were statistically significant differences in the prevalences obtained, the chi square or Fisher exact test was used. In addition, the prevalence of the 16 symptoms included in the CVS-Q ${ }^{\odot}$ was calculated.

To measure the association between CVS and the variables related to health, age, sex, optical correction and VDT exposure, logistic regression models were carried out. The measure of association obtained was the odds ratio (OR), crude (cOR) and adjusted (aOR) for all the explanatory variables studied with $95 \%$ confidence intervals $(95 \% \mathrm{CI})$. A p- value < 0.05 was considered statistically significant. The statistical program SPSS version 25 was used for the analyses.

\section{Results}

Table 1 shows the description of the sample. The mean age was 20.7 ( $S D=2.1)$, with a range between 18 and 29 years. Fifty seven percent were women. Only 4.5\% presented with ocular disorders, the most frequent being strabismus $(n=5)$ and amblyopia $(n=4)$, all of them different subjects. A total of $25.8 \%$ of the sample was undergoing general pharmacological treatment, mainly contraceptive pills $(n=39)$, antihistamines $(n=8)$ and antibiotics $(n=6)$. $1.6 \%$ was receiving ocular pharmacological treatment to treat ocular hypertension, allergic conjunctivitis or was taking vitamins for retinal health. In addition, $1.6 \%$ had undergone some ocular surgery (for strabismus, chalazion or lacrimal duct). A total of $62.3 \%$ used glasses, of those the $52.6 \%$ also used contact lenses (16.4\% used contact lenses daily and 
17.2\% used them occasionally on weekends and/or for sports). Two participants who used ortho-k contact lenses did not wear glasses. Among the people who used optical correction, glasses were used more often than contact lenses to study. However, 14 students did not use optical correction to study but did so for other activities. This could be due to the fact that low-moderate myopes are quite likely to take their glasses off for studying. The average hours of VDT use to study was 3.2 ( $\mathrm{SD}=2.1$ ), with a range between 0 and 10 hours a day, and only $32.8 \%$ of the participants took scheduled breaks to rest their eyes. The average duration of these breaks was 6.7 minutes $(\mathrm{SD}=15.4)$. For reasons other than to study, $61.5 \%$ of the participants used VDTs between 4 and 8 hours a day, and 15.6\% used them more than 8 hours a day. The average total VDT use was 9.0 hours $(\mathrm{SD}=3.7)$, with a range between 2 and 22 hours per day.

The total prevalence of CVS was 76.6\%, with a mean score of 8.1 (SD = 3.4) points on the CVS-Q ${ }^{\odot}$. Statistically significant differences were observed in the prevalence of CVS by sex, age, regular use of glasses, use of optical correction to study, hours of VDT use to study and hours of total VDT use (Table 2). The most frequent symptoms were headache $(78.7 \%)$, itching (73.0\%), heavy eyelids (61.9\%), and feeling that sight is worsening $(60.3 \%)$; the least frequent symptoms were colored halos around objects $(21.3 \%)$ and double vision (8.6\%). As Figure 1 shows, almost all symptoms occurred occasionally in the majority of cases. However, headache, heavy eyelids and dryness were often or always present in more than $15 \%$ of the sample analyzed. In general, the intensity of symptoms was moderate; the exception was headaches, which more than $27 \%$ of the students reported were intense.

As Figure 2 shows, the association with CVS was lower among the older students (22-29 years) than among the youngest students (18-19 years). Likewise, CVS was higher in women than in men, in those who usually wore glasses compared to those who did not, and in those who wore glasses to study compared to those who wore neither glasses nor contact lenses for this purpose. Regarding VDT use, among students who used VDTs more than 2 hours per day to study and those who used them more than 4 hours per day in total (for study and leisure), the association with CVS was higher. The results of the multivariate analysis indicated two factors that were associated with CVS in the proposed model: use of VDTs for more than 4 hours a day to study, which was associated with an increased likelihood of CVS compared with VDT use for less than 2 hours a day (84.2\% vs. 62.3\%; aOR = 3.428), and being between 22-29 years of age, which was associated with a decreased likelihood of CVS compared with the youngest group $(67.1 \%$ vs. $84.7 \%$; aOR $=0.360)$. Note that Figure 2 
shows the results for those variables with statistically significant results of the simple logistic regression (collected for all the study variables in Appendix).

\section{Discussion}

The results of this study estimate a CVS prevalence greater than $75 \%$ in the Spanish university population, with headache, itching eyes, heavy eyelids and feeling that sight is worsening as the most frequent symptoms. In the crude analysis, it was observed that being a woman, using glasses daily and use for study rather than leisure, and more hours of VDT use, in general, are associated with an increase in CVS, while age acts as a protective factor. However, after adjusting for all the variables studied, the increase in CVS is explained only by the use of VDTs to study for more than 4 hours a day, while the decrease in the syndrome is related to an age between 22 and 29 years.

The prevalence of CVS observed in the present study is high and close to values obtained in other studies in the university population, although those studies used measurement instruments and definitions of CVS that differed from ours. As an example, the study by Kharel et al., [6] obtained a CVS prevalence of $71.6 \%$ in medical students at a university in Nepal who responded to a questionnaire on 8 symptoms related to CVS (neck pain, headache, eyestrain, double and blurred vision, dryness, watery eyes and ocular redness); in that study, the presence of at least one of these symptoms was considered indicative of CVS. Additionally, $37.2 \%$ of students in that study spent 2 to 3 hours a day in front of a computer (the tasks for which it was used were note specified), while in our study, only the average use of VDTs to study exceeded this value, and the mean global duration of VDT use was much higher (9.0 hours/day). In particular, given that the CVS-Q ${ }^{\odot}$ evaluates 16 symptoms and given the very high amounts of VDT use among our students, we believe that the differences in prevalence between our study and that of Kharel et al., should have been more evident. The same was observed with other studies from Asia that estimate an even higher prevalence of CVS than we found in our study, despite lower exposure to VDTs and the evaluation of fewer symptoms $[3,18]$. In future studies, it would be interesting to compare the prevalence of CVS in university students from different countries using a similar methodological procedure and to analyze which factors (individual, environmental, socioeconomic, or other) influence the differences found.

Similarly, when our prevalence result is compared with the results of studies of the working population that measure CVS with the same instrument $\left(\mathrm{CVS}-\mathrm{Q}^{\odot}\right)$, the prevalence of 
CVS in university students appears to be much higher. Thus, in the study by Tauste et al., [20] a CVS prevalence of 53\% was obtained in a sample of administrative personnel from the province of Alicante (Spain), and in the study by Seguí-Crespo et al., [24] the prevalence was $62.5 \%$ in Italian workers who were exposed to VDTs in their work. These differences in the prevalence of CVS among workers and students may occur because the student population grew up at the height of the NICT use, which could have a cumulative effect on eye health in current generations of young people who have spent an exorbitant amount of time using VDTs since childhood [25].

Regarding symptomatology, headache is one of the most often reported symptoms in other studies in the university population. Al Tawil et al., [5] estimated a headache prevalence of $66.5 \%$, indicating that it was the most prevalent symptom. Other studies found differences by sex; in the study by Mowatt et al., [2] the prevalence of headache in men was $36.6 \%$, while that in women was 59.6\% $(P=0.001)$. Shantakumari et al., [1] also reported that headache was the most frequent symptom, with differences between women and men (58.2\% vs. $43.9 \%, P=0.03$ ). In our study, headache was also the most prevalent symptom; furthermore, $20.1 \%$ of the sample often or always suffered from it, and $27.5 \%$ perceived it intensely. The prevalence in women was $87.1 \%$, and in men was $67.6 \%(P<0.001)$, which indicates that our findings are in line with those presented by these authors.

However, although many studies indicate that women and men suffer more from different symptoms, in university students, the association of sex with CVS is not clear [18], which is consistent with our results. Nevertheless, it is a far contrast with the results obtained for the working population, in which CVS occurs more frequently in women [20,26]. Authors such as Rahman et al., [27] and Toomingas et al., [28] observed that women workers are twice as likely to suffer from visual symptomatology due to the use of VDT than men (aOR = 2.30; $95 \% \mathrm{CI}=1.45-3.65$ and $\mathrm{aOR}=1.78 ; 95 \% \mathrm{CI}=1.35-2.34$, respectively). We believe that the influence of hormonal factors in young women students and in women workers of perimenopausal and menopausal age could partly explain these discrepancies, with more ocular and visual symptomatology occurring in the latter group $[29,30]$.

In the present study, we observed that longer durations of VDT use to study are associated with CVS. Specifically, the use of VDTs for this purpose for more than 4 hours per day carries a 3 times higher likelihood of CVS than using VDTs for less than 2 hours. On the other hand, no association has been observed between CVS and the global duration of exposure to VDTs, which includes the use of digital devices for both study and leisure. There 
are several studies in university students that have also demonstrated the relationship between the time of use of VDT and an increase in the symptoms of CVS but do not specify whether this time is dedicated to tasks related to the study or other activities. In two of these studies, a higher prevalence of symptoms was observed in university students who used the computer more than 2 hours a day $(P<0.001)[3,6]$. Another study estimated that the use of electronic devices for more than 5 hours a day was positively associated with experiencing more than three symptoms of CVS (OR $=1.52 ; 95 \% \mathrm{CI}=1.07-2.16)$ [5]. In our case, the fact that the use of digital devices to study is more strongly related to symptomatology than using them for activities related to leisure could be explained because different activities might entail different intensity and duration of visual demands, subsequently impacting CVS [31]. The cognitive demands may also be different and less-demanding cognitive tasks carry a lower risk of experiencing CVS [32]. One could even assume that the type of devices used for leisure are dissimilar to those used for study (hand-held devices vs. desktop/laptop computers). On the other hand, one possible difficulty in studying this topic is that students may not be properly estimating the time they spend using VDTs. Today, the almost continuous use of digital devices makes it difficult to adequately quantify and determine the time allocated to each task. If we observe the maximum VDT exposure in our study, we notice that they are excessively high (some people indicated spending more than 20 hours a day in front of a screen). For the future, tools are needed that can methodologically help us to objectify exposure, since only in this way can the association between CVS and the duration of VDT use be reliably analyzed.

Additionally, in the present study, no association with CVS was observed for wearing glasses, contact lenses, or both, either routinely or to study compared to not using optical correction. Those who did not use optical correction (glasses and/or contact lenses) to study were emmetropes, probably low myope and those who used ortho-k contact lenses. Although several studies have observed that using glasses increases the probability of suffering CVS in the university population $[3,7,18]$, none have evaluated the existence of possible uncorrected refractive errors. Regarding the use of contact lenses, in contrast with our results, the study of Tauste et al., [20] found that workers who used VDTs more than 6 hours a day and were contact lens wearers had a higher probability of suffering CVS $(\mathrm{OR}=4.85 ; 95 \% \mathrm{CI}=1.25$ 18.80). These differences may be due to students having better tear and ocular surface conditions, which facilitate adaptation to contact lenses, compared to older workers, who have a higher rate of problems with ocular dryness. However, according to our results, the prevalence of CVS decreases with age. These results agree with those obtained in the study 
carried out by Rahman et al., who found after adjusting for all variables that the youngest group had a 3-fold higher probability of suffering CVS ( $\mathrm{aOR}=2.89 ; 95 \% \mathrm{CI}=1.38-6.04)$ [27]. Therefore, more studies are needed to explain the effects of age on CVS in university students, and these studies should incorporate objective clinical tests of the students' ocular health and refractive status to provide information about their ocular conditions, the state of their vision and whether they have adequate optical correction.

Regarding the limitations of the present study, the sample was not randomly selected, but it was a heterogeneous sample composed of students from 44 different degree programs and representative of the population under study in terms of sample size. Additionally, as mentioned above, it is possible that the students overestimated their exposure time to digital devices (especially the hours of use dedicated to leisure). This study could be even more complete if it had included a clinical exploration of the participants and had evaluated other parameters that could have an effect, such as the type of digital device or the ergonomic conditions during VDT use. Even so, this is the first study of the university population in Spain in which a validated instrument was used to estimate the prevalence of CVS, which is its main strength.

Ultimately, this study aims to be a consistent starting point that makes visible the high prevalence of ocular and visual symptomatology that Spanish university students, particularly the youngest and those who use VDTs to study for longer durations, experience in their dayto-day lives in relation to the use of digital devices. As is known, CVS is a health problem that can decrease student performance [9]; consequently, and given the high prevalence of CVS found (76.6\%), it is essential to continue investigating its causes and thus to establish the preventive measures necessary to reduce it. On the other hand, given that the influence of type of task is probably one of the least explored areas of research in the CVS literature [31], activities involving VDTs that require high concentration and intense visual demands should be considered, especially when studying CVS in young adults.

Public awareness of the CVS in the university environment should be highlighted among students, teachers and the administrators. The universities could carry out preventive strategies such as conducting sensitization campaigns aimed at young people to provide information on the implications of this syndrome. It would be ideal to educate university students and instill in them right practice of using digital devices. Remedial measures to reduce the symptoms of CVS [11] especially during prolonged use of these devices to study should be followed by students. 


\section{References}

[1] N. Shantakumari, R. Eldeeb, J. Sreedharan, K. Gopal, Computer use and vision-related problems among university students in Ajman, United Arab Emirate. Ann. Med. Health. Sci. Res. 4 (2014) 258-63. doi: 10.4103/2141-9248.129058

[2] L. Mowatt, C. Gordon, ABR. Santosh, T. Jones, Computer vision syndrome and ergonomic practices among undergraduate university students. Int. J. Clin. Pract. 72 (2018) 1-7. doi: 10.1111/ijcp.13035

[3] SC. Reddy, CK. Low, YP. Lim, LL. Low, F. Mardina, MP. Nursaleha, Computer vision syndrome: a study of knowledge and practices in university students. Nepal. J. Ophtalmol. 5 (2013) 161-8. doi: 10.3126/nepjoph.v5i2.8707

[4] SH. Al Rashidi, H. Alhumaidan, Computer vision syndrome prevalence, knowledge and associated factors among Saudi Arabia University Students: Is it a serious problem? Int. J. Health. Sci. (Qassim). 11 (2017) 17-1. PMID: 29114189

[5] L. Al Tawil, S. Aldokhayel, L. Zeitouni, T. Qadoumi, S. Hussein, SS. Ahamed, Prevalence of self-reported computer vision syndrome symptoms and its associated factors among university students. Eur. J. Ophthalmol. 30 (2020) 189-95. doi: $10.1177 / 1120672118815110$

[6] R. Kharel, A. Khatri, Knowledge, Attitude and practice of Computer Vision Syndrome among medical students and its impact on ocular morbidity. J. Nepal. Health. Res. Counc. 16 (2018) 291-6. PMID: 30455488

[7] HR. Moldovan, ST. Voidazan, G. Moldovan, MA. Vlasiu, G. Moldovan, R. Panaitescu, Accommodative asthenopia among Romanian computer-using medical students- A neglected occupational disease. Arch. Environ. Occup. Health. 75 (2020) 235-41. doi: $10.1080 / 19338244.2019 .1616666$

[8] J. Tondeur, I. Sinnaeve, M. van Houtte, J. van Braak, ICT as cultural capital: The relationship between socioeconomic status and the computer-use profile of young people. New. Med. Soc. 13 (2011) 151-68. doi: 10.1177/1461444810369245

[9] AL. Sheppard, JS. Wolffsohn, Digital eye strain: prevalence, measurement and amelioration. BMJ. Open. Ophthalmol. 3 (2018) e000146. doi: 10.1136/bmjophth-2018000146 
[10] C. Coles-Brennan, A. Sulley, G. Young, Management of digital eye strain. Clin. Exp. Optom. 102 (2019) 18-29. doi: 10.1111/cxo.12798

[11] S. Munshi, A. Varghese, S. Dhar-Munshi, Computer vision syndrome - A common cause of unexplained visual symptoms in the modern era. Int. J. Clin. Pract. 71 (2017) 15. doi: 10.1111/ijcp.12962

[12] American Optometric Association, Computer Vision Syndrome. https://www.aoa.org/patients-and-public/caring-for-your-vision/protecting-yourvision/computer-vision-syndrome. Accessed April 25,2020

[13] C. Blehm, S. Vishnu, A. Khattak, S. Mitra, RW. Yee, Computer Vision Syndrome: A Review. Surv. Ophthalmol. 50 (2005) 253-62. doi: 10.1016/j.survophthal.2005.02.008

[14] M. Rosenfield, Computer vision syndrome: a review of ocular causes and potential treatments. Ophthalmic. Physiol. Opt. 31 (2011) 502-15. doi: 10.1111/j.14751313.2011.00834.X

[15] WD. Thomson, Eye problems and visual display terminals - the facts and the fallacies. Ophthalmic. Physiol. Opt. 18 (1998) 111-9. PMID: 9692030

[16] C. Salibello, E. Nilsen, Is there a typical VDT patient? A demographic analysis. J. Am. Optom. Assoc. 66 (1995) 479-83. PMID: 7494082

[17] A. Sen, S. Richardson, A study of computer-related upper limb discomfort and computer vision syndrome. J. Hum. Ergol. (Tokyo). 36 (2007) 45-50. PMID: 18572794

[18] M. Logaraj, V. Madhupriya, S. Hegde, Computer vision syndrome and associated factors among medical and engineering students in Chennai. Ann. Med. Health. Sci. Res. 4 (2014) 179-85. doi: 10.4103/2141-9248.129028

[19] MDM. Seguí, J. Cabrero-García, A. Crespo, J. Verdú, E. Ronda, A reliable and valid questionnaire was developed to measure computer vision syndrome at the workplace. J. Clin. Epidemiol. 68 (2015) 662-73. doi: 10.1016/j.jclinepi.2015.01.015

[20] A. Tauste, E. Ronda, MJ. Molina, M. Seguí, Effect of contact lens use on Computer Vision Syndrome. Ophthalmic. Physiol. Opt. 36 (2016) 112-9. doi: 10.1111/opo.12275

[21] M. Sánchez-Brau, B. Domenech-Amigot, F. Brocal-Fernández, JA. Quesada-Rico, M. Seguí-Crespo, Prevalence of Computer Vision Syndrome and its relationship with 
ergonomic and individual factors in presbyopic VDT workers using progressive addition lenses. Int. J. Environ. Public. Health. 17 (2020) 1003. doi: 10.3390/ijerph17031003

[22] EM. Artime Ríos, F. Sánchez Lasheras, A. Suarez Sánchez, FJ. Iglesias-Rodríguez, MDM. Seguí Crespo, Prediction of Computer Vision Syndrome in health personnel by means of generic algorithms and binary regression trees. Sensors (Basel). 19 (2019) 2800. doi: $10.3390 / \mathrm{s} 19122800$

[23] EM. Artime Ríos, A. Suárez Sánchez, F. Sánchez Lasheras, M. Seguí Crespo, Genetic algorithm based on support vector machines for computer vision syndrome classification in health personnel. Neural. Comput. Appl. 32 (2018) 1239-48. doi: 10.1007/s00521018-3581-3

[24] MDM. Seguí-Crespo, N. Cantó Sancho, E. Ronda, R. Colombo, S. Porru, A. Carta, Translation and cultural adaptation of the Computer Vision Syndrome Questionnaire (CVS-Q) into Italian. Med. Lav. 110 (2019) 37-45. doi: 10.23749/mdl.v110i1.7499

[25] A. Sigman, Time for a view on screen time. Arch. Dis. Child. 97 (2012) 935-42. doi:10.1136/archdischild-2012-302196

[26] P. Ranasinghe, WS. Wathurapatha, YS. Perera, DA. Lamabadusuriya, S. Kulatunga. N. Jayawardana, P. Katulanda, Computer vision syndrome among computer office workers in a developing country: an evaluation of prevalence and risk factors. BMC. Res. Notes. 9 (2016) 150. doi: 10.1186/s13104-016-1962-1

[27] ZA. Rahman, S. Sanip, Computer user: demographic and computer related factors that predispose user to get computer vision syndrome. IJBHT. 1 (2011) 84-91

[28] A. Toomingas, M. Hagberg, M. Heiden, H. Richter, KE. Westergren, EW. Tornqvist. Risk factors, incidence and persistent of symptoms from the eyes among professional computer users. Work. 47 (2014) 291-301. doi: 10.3233/WOR-131778

[29] DA. Sullivan, EM. Rocha, P. Aragona, JA. Clayton, J. Ding, B. Golebiowski, U. Hampel, AM. McDermott, DA. Schaumberg, TFOS DEWS II Sex, Gender, and Hormones Report. Ocul. Surf. 15 (2017) 284-333. doi: 10.1016/j.jtos.2017.04.001

[30] T. Peck, L. Olsakovsky, S. Aggarwal, Dry eye syndrome in menopause and perimenopausal age group. J. Midlife. Health. 8 (2017) 51-4. doi: 10.4103/jmh.JMH_41_17 
[31] Z. Yan, L. Hu, H. Chen, F. Lu, Computer Vision Syndrome: A widely spreading but largely unknown epidemic among computer users. Comput. Human. Behav. 24 (2008) 2026-42. doi: 10.1016/j.chb.2007.09.004

[32] J. Anshel, Visual ergonomics handbook. 1st ed. New York, NY: Taylor \& Francis; 2005.

\section{Figure legends}

Figure 1. Percentage of university students who presented each symptom according to frequency and intensity.

Figure 2. Association between computer vision syndrome (CVS) and sociodemographic variables, optical correction and video display terminal (VDT) exposure: crude (cOR) and adjusted (aOR) odds ratio, 95\% confidence intervals (95\% CI) and P value. CL: contact lenses. 
Table 1. Distribution of the sample $(n=244)$ of the university population according to sociodemographic, ocular health, optical correction characteristics and exposure to digital devices.

Variables

Total

n $\%$

Sex

Male

Female

Age (years)

$$
\text { 18-19 }
$$

20-21

22-29

Ocular disorders

No

Yes

11

$105 \quad 43.0$

139

57.0

72

102

70

28.7

No

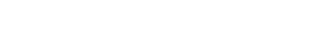


$C L$

Both

Use of VDT to study (hours/day)

$<2$

2-4

$>4$

Scheduled breaks during the study

$$
\text { No }
$$$$
\text { Yes }
$$

164

80

56

$150 \quad 61.5$

38

15.6

Total use of VDT (hours/day)

$$
\leq 4
$$

$>4-8$

$>8$

11

4.5

3

1.2

53

$134 \quad 54.9$

$57 \quad 23.4$

$64 \quad 67.2$

32.8

Use of VDT to leisure (hours/day)

$4-8$
$>8$

$24 \quad 9.8$

$94 \quad 38.5$

$126 \quad 51.6$

CL: contact lenses; VDT: video display terminal. 
Table 2. Prevalence of computer vision syndrome (CVS) and differences according to the variables studied.

\begin{tabular}{|c|c|c|c|}
\hline Variables & $\mathrm{n}$ & $\%$ & $\mathrm{P}$ value \\
\hline Total & 187 & 76.6 & \\
\hline \multicolumn{4}{|l|}{$\operatorname{Sex}^{\dagger}$} \\
\hline Male & 73 & 69.5 & \multirow[t]{2}{*}{$0.022 *$} \\
\hline Female & 114 & 82.0 & \\
\hline \multicolumn{4}{|l|}{ Age $(\text { years })^{\dagger}$} \\
\hline $18-19$ & 61 & 84.7 & \multirow[t]{3}{*}{$0.045^{*}$} \\
\hline $20-21$ & 79 & 77.5 & \\
\hline $22-29$ & 47 & 67.1 & \\
\hline \multicolumn{4}{|c|}{ Ocular disorders $*$} \\
\hline No & 179 & 76.8 & \multirow[t]{2}{*}{0.722} \\
\hline Yes & 8 & 72.7 & \\
\hline \multicolumn{4}{|c|}{ General pharmacological treatment ${ }^{\dagger}$} \\
\hline No & 134 & 74.0 & \multirow[t]{2}{*}{0.103} \\
\hline Yes & 53 & 84.1 & \\
\hline \multicolumn{4}{|c|}{ Ocular pharmacological treatment } \\
\hline No & 184 & 76.7 & \multirow[t]{2}{*}{1.000} \\
\hline Yes & 3 & 75.0 & \\
\hline \multicolumn{4}{|l|}{ Ocular surgery ${ }^{\ddagger}$} \\
\hline No & 184 & 76.7 & \multirow[t]{2}{*}{1.000} \\
\hline Yes & 3 & 75.0 & \\
\hline \multicolumn{4}{|l|}{ Use of glasses $^{\dagger}$} \\
\hline No & 61 & 66.3 & \multirow[t]{2}{*}{$0.003 * *$} \\
\hline Yes & 126 & 82.9 & \\
\hline \multicolumn{4}{|c|}{ Use of contact lenses ${ }^{\dagger}$} \\
\hline No & 120 & 74.1 & \multirow[t]{2}{*}{0.183} \\
\hline$Y e s$ & 67 & 81.7 & \\
\hline \multicolumn{4}{|c|}{ Use of optical correction to study } \\
\hline No & 72 & 67.9 & \multirow[t]{3}{*}{$0.038 *$} \\
\hline Glasses & 104 & 83.9 & \\
\hline$C L$ & 9 & 81.8 & \\
\hline
\end{tabular}

This article is protected by copyright. All rights reserved 
Use of VDT to study (hours/day) ${ }^{\dagger}$

$$
\begin{aligned}
& <2 \\
& 2-4 \\
& >4
\end{aligned}
$$

106

48

64

41

117

29

14

75

98

$$
\text { No }
$$

$$
\begin{aligned}
& <4 \\
& 4-8 \\
& >8
\end{aligned}
$$

$$
\begin{aligned}
& \leq 4 \\
& >4-8 \\
& >8
\end{aligned}
$$

62.3

$0.015^{*}$

79.1

84.2

Scheduled breaks during the study ${ }^{\dagger}$

Use of VDT to leisure (hours/day) ${ }^{\dagger}$

Total use of VDT (hours/day) ${ }^{\dagger}$

CL: contact lenses; VDT: video display terminal.

$\uparrow$ Chi-square.

† Fisher exact test.

$* P<0.05 ; * * P<0.01$.
58.3

79.8

77.8
73.2

76.3

5.0

80.0

3.2
7.769

.0

0.078

87.8

0.386 
Figure 1. Percentage of university students who presented each symptom according to frequency and intensity.

Frequency

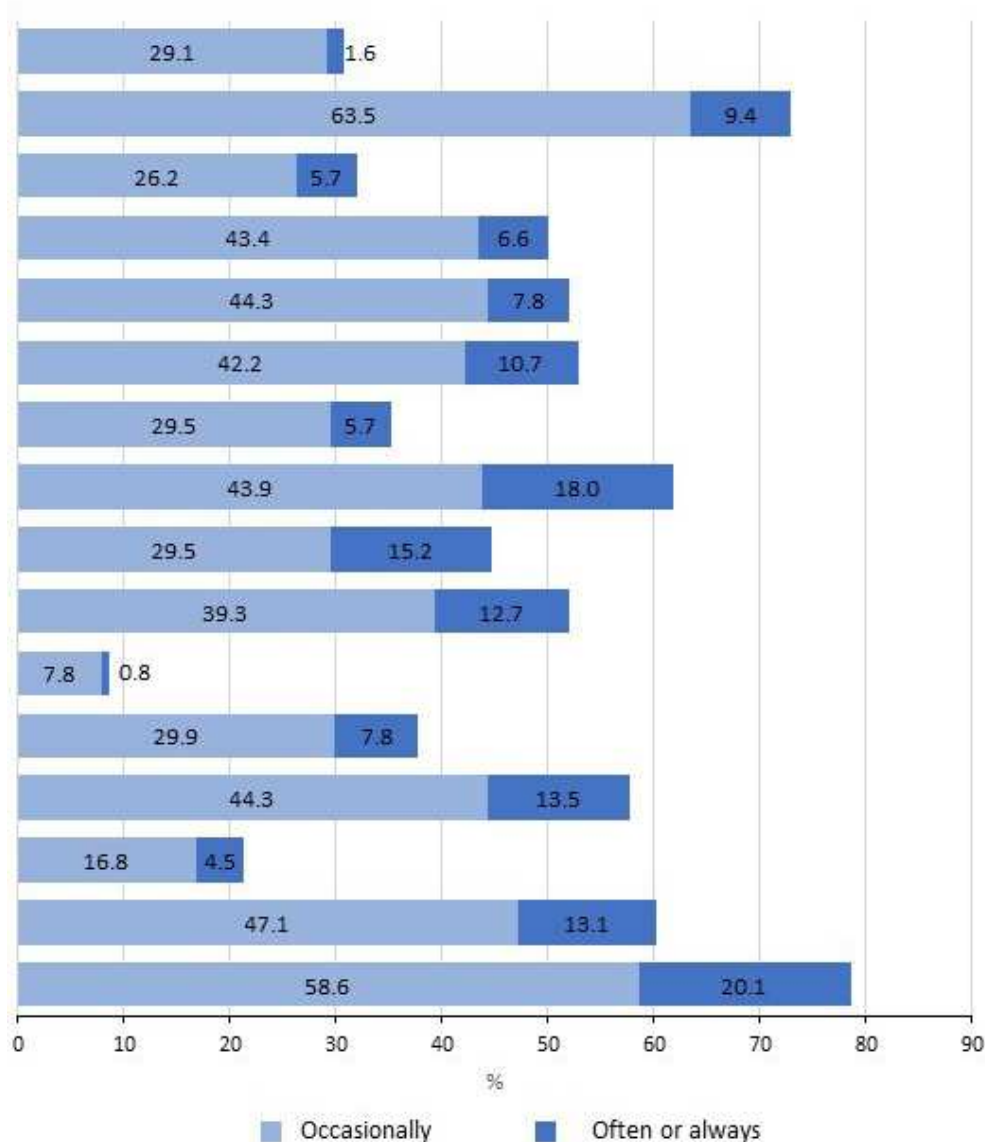

Intensity

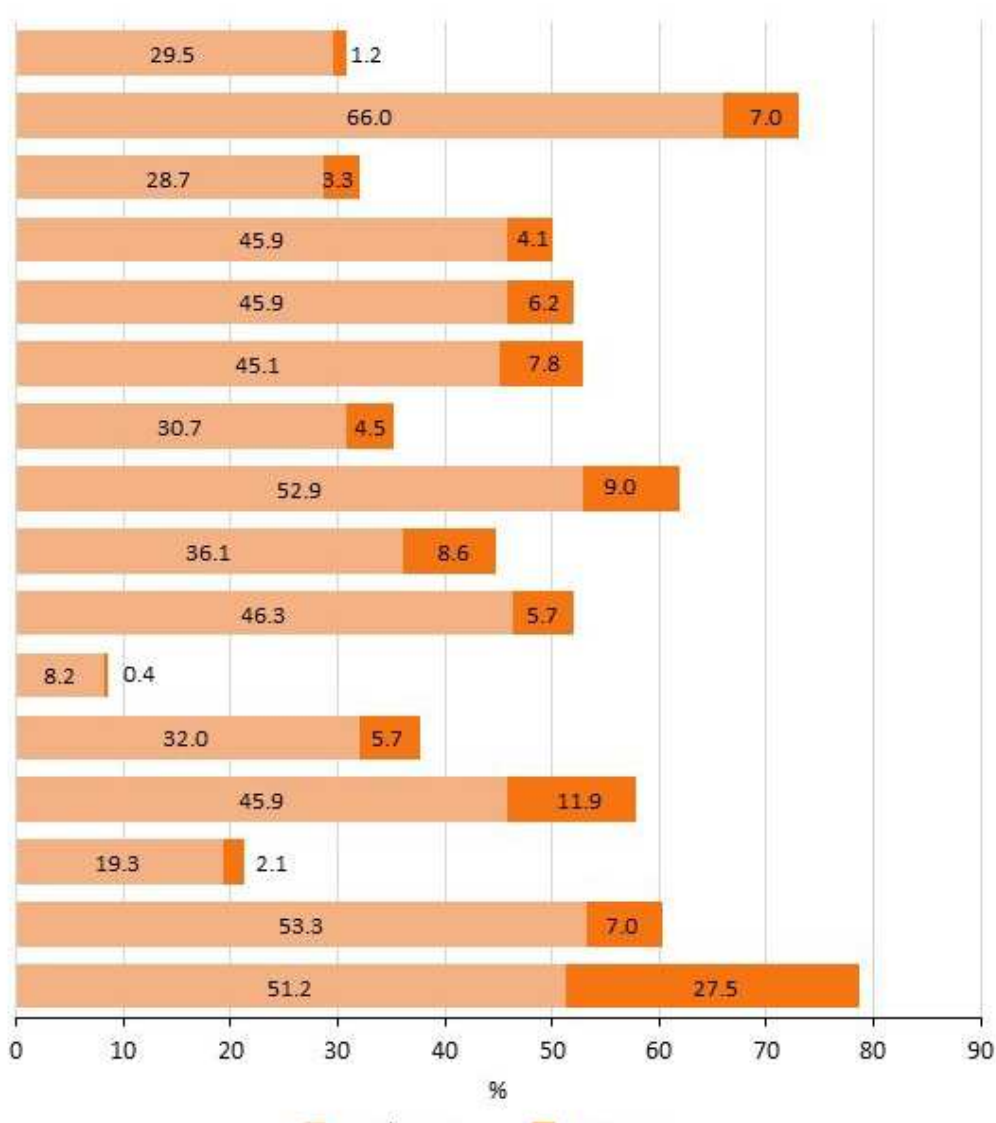

Moderate 
Figure 2. Association between computer vision syndrome (CVS) and sociodemographic variables, optical correction and video display terminal (VDT) exposure: crude (cOR) and adjusted (aOR) odds ratio, 95\% confidence intervals $(95 \% \mathrm{CI})$ and P value. CL: contact lenses.

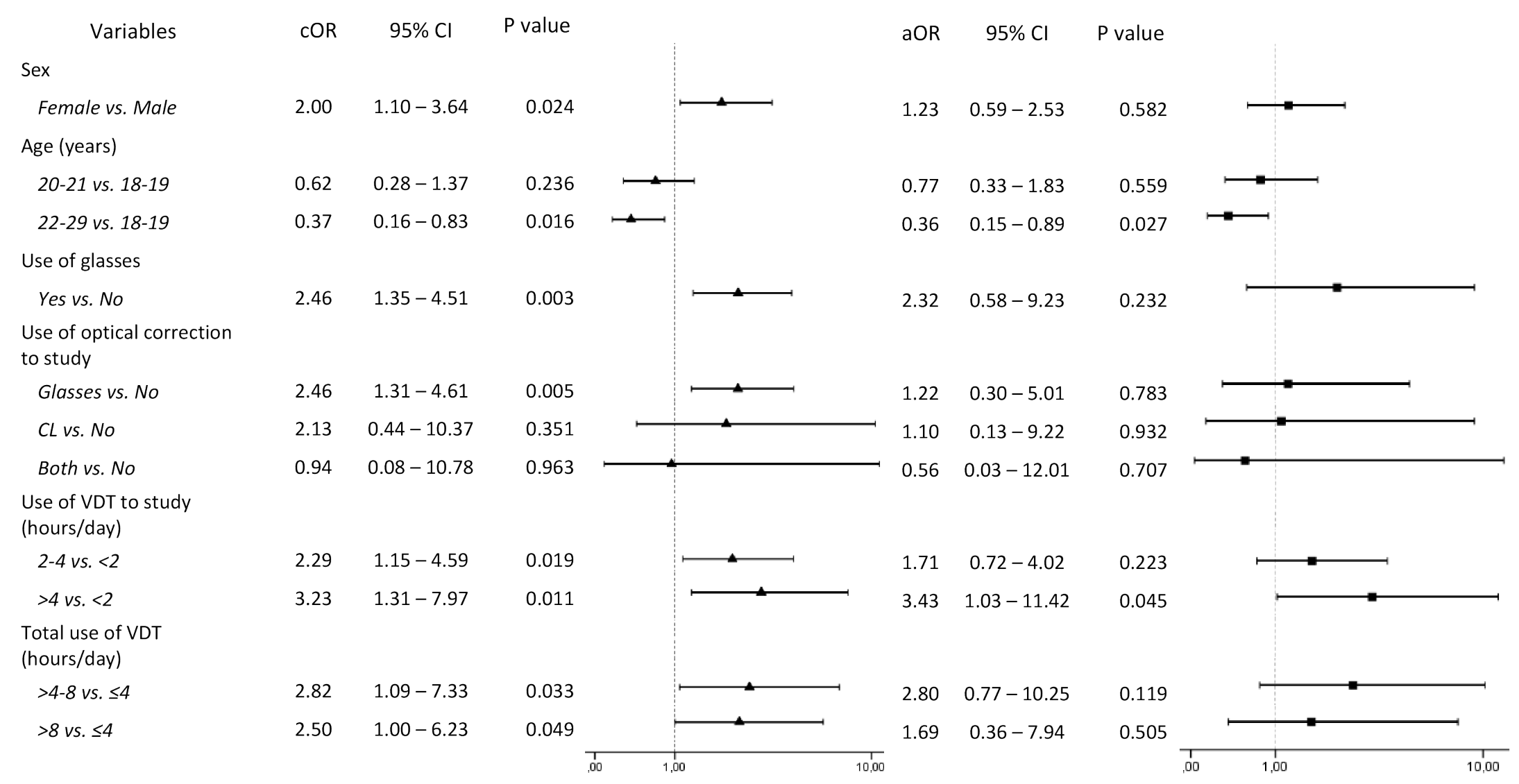

